\title{
YWHAE as an HE4 interacting protein can influence the malignant behaviour of ovarian cancer by regulating the PI3K/AKT and MAPK pathways
}

Xiao Li, Caixia Wang, Shuang Wang, Yuexin Hu, Shan Jin, Ouxuan Liu, Rui Gou, Xin Nie, Juanjuan Liu and Bei Lin* (1)

\begin{abstract}
Background: Malignant tumours of the female reproductive system threaten the lives and health of women worldwide, with ovarian cancer having the highest mortality rate. Based on previous work, this study analysed the expression and role of YWHAE in ovarian epithelial tumours.

Methods: The interaction between YWHAE and HE4 was evaluated via immunoprecipitation, western blot analysis, and cellular immunofluorescence. Immunohistochemistry was used to address the relationship between YWHAE expression, clinicopathological parameters, and patient prognosis. Changes in cell invasion, epithelial-mesenchymal transition, migration, proliferation, apoptosis, and cell cycle before and after differential expression of YWHAE were also explored in ovarian cancer cell lines and via in vivo experiments.

Results: YWHAE was found to interact with HE4, and its expression was positively correlated with HE4 expression. Moreover, YWHAE upregulation was associated with advanced stages of ovarian cancer and poor patient prognosis. In addition, YWHAE enhanced invasion, migration, and proliferation, but inhibited the apoptosis of ovarian cancer cells. These biological effects were found to be mediated by the AKT and MAPK signalling pathways.
\end{abstract}

Conclusions: Altogether, this study demonstrates that YWHAE is substantially upregulated in ovarian cancer tissues, representing a risk factor for the prognosis of ovarian cancer that is positively correlated with HE4 expression. Furthermore, YWHAE and its downstream pathways may represent new therapeutic targets for ovarian cancer.

Keywords: YWHAE, HE4, Interacting protein, Poor prognosis, Malignant behaviour, Ovarian cancer

\section{Introduction}

Ovarian cancer is among the most common malignant tumours of the female reproductive system and has the highest mortality rate. Due to the lack of obvious or specific symptoms, or reliable screening methods, rates

*Correspondence: linbei88@hotmail.com

Department of Obstetrics and Gynecology, Shengjing Hospital Affiliated to China MedicalUniversity, No. 36, Sanhao Street, Heping District,

Shenyang 110004, People's Republic of China of early detection and diagnosis of ovarian cancer are extremely low [1], resulting in advanced tumor progression, and shortened tumour-free and overall survival times [2]. Therefore, there is an urgent need to identify tumour markers with high sensitivity and specificity to guide early clinical screening and diagnosis and for monitoring ovarian cancer.

Human epididymis protein 4 (HE4) is a highly sensitive and specific ovarian cancer marker that has been identified via genomics and proteomics screenings [3].

(c) The Author(s) 2021. This article is licensed under a Creative Commons Attribution 4.0 International License, which permits use, sharing, adaptation, distribution and reproduction in any medium or format, as long as you give appropriate credit to the original author(s) and the source, provide a link to the Creative Commons licence, and indicate if changes were made. The images or other third party material in this article are included in the article's Creative Commons licence, unless indicated otherwise in a credit line to the material. If material is not included in the article's Creative Commons licence and your intended use is not permitted by statutory regulation or exceeds the permitted use, you will need to obtain permission directly from the copyright holder. To view a copy of this licence, visit http://creativeco mmons.org/licenses/by/4.0/. The Creative Commons Public Domain Dedication waiver (http://creativecommons.org/publicdomain/ zero/1.0/) applies to the data made available in this article, unless otherwise stated in a credit line to the data. 
Researchers have shown that HE4 detection is more advantageous than the detection of the common tumour marker CA125 for the early diagnosis of ovarian cancer and for monitoring disease progression [4]. In 2003, HE4 was designated as a serum marker for ovarian cancer and was approved by the U.S. Food and Drug Administration in 2009 for monitoring the recurrence and progression of epithelial ovarian cancer [5]. Currently, several ongoing clinical studies are addressing the diagnostic potential of HE4; however, few are exploring its underlying molecular mechanisms.

Our previous study has demonstrated that HE4 is highly expressed in ovarian cancer tissues, and that the interaction between HE4 and annexin A2 promotes the invasion and metastasis of ovarian cancer. This is accomplished by activating adhesion signalling pathways, such as mitogen-activated protein kinase (MAPK) and FOCAL $[6,7]$. Proteins perform their biological functions primarily by forming protein complexes, or by working with chaperone molecules. The coordination of proteins with different functions constitutes the basic process of life. Therefore, given the crucial role of HE4 in ovarian cancer, it is clear that recognition and improved knowledge on HE4-interacting proteins is an important step for a better understanding of this disease. A previous study using HE4 as bait in a two-hybrid screen identified the tyrosine 3-monooxygenase/tryptophan 5-monooxygenase activation protein epsilon (YWHAE) protein as a partner for HE4.

YWHAE, also known as $14-3-3 \varepsilon$, belongs to the YWHA protein family, which comprises at least seven highly conserved subtypes of soluble acidic proteins encoded by different genes, namely $\beta, \varepsilon, \eta, \gamma, \tau, \xi$, and $\sigma$ [8]. It can be widely combined with other proteins such as kinases, phosphatases, transmembrane receptors, and transcription factors, among other target proteins [9-11], to function as a protein interaction bridge in a wide range of biological processes [12-14].

X-ray diffraction analysis showed that YWHAE protein monomers form homodimers or heterodimers [15] that are bound by highly conserved hydrophobic amino acids. Different YWHA subtypes can bind to the same target, giving these proteins the ability to regulate many physiological processes, including cell proliferation, apoptosis, protein transport, metabolic regulation, and signal transduction, among others $[16,17]$. The structure of YWHAE may be the basis for its role as a "bridge protein", as well as its contribution towards disease incidence [18].

Based on previous preliminary findings, this study explored the relationship between YWHAE and HE4 and analysed the expression of YWHAE in ovarian epithelial tumours, as well as its mechanism of action. Improved knowledge regarding the activity of YWHAE and HE4 may provide a basis to further explore the development and progression of ovarian cancer and to design novel diagnostic strategies.

\section{Materials and methods Primary samples}

Ovarian tissue samples were surgically collected between 2008 and 2012 from patients at the Department of Obstetrics and Gynecology, Shengjing Hospital, China Medical University. A total of 16 samples were derived from normal ovarian tissue removed due to uterine fibroids or cervical cancer (normal group), 18 samples were from benign cases, 24 samples were classified as borderline, and 105 samples were from malignant ovarian cancers. The pathological types among the malignant samples included 71 serous tumours, seven mucinous tumours, 19 endometrioid tumours, and eight clear cell carcinomas. The malignant group was also classified according to pathology assessment, with 51 cases identified as highly differentiated and 54 as poorly differentiated. Surgical pathological staging was performed in accordance with the International Federation of Obstetrics and Gynecology (FIGO) standards: 44 cases were in stage I-II and 61 were in stage III-IV. A comprehensive exploratory surgery was performed in stage I-II, and cytoreductive surgery was performed in stage III-IV. In the malignant group, 92 patients underwent lymph node dissection, with lymph node metastasis being confirmed in 28 cases. All cases were newly diagnosed, and patients were radiotherapy- and chemotherapy-naïve.

\section{Immunochemistry}

Histopathological specimens were fixed with $10 \%$ formalin solution, embedded in paraffin, and then serially sectioned into $5 \mu \mathrm{m}$ slices. The paraffin sections were deparaffinised with xylene and re-hydrated with gradient alcohol solutions, and the antigens were recovered by heating. Subsequently, $\mathrm{H}_{2} \mathrm{O}_{2}$, goat serum blocking solution, and anti-YWHAE antibody (1:100, sc-23957, Santa Cruz Biotechnology, Santa Cruz, CA) or an antiHE4 antibody (1:1500, ab200828, Abcam, Cambridge, UK) were sequentially added in a dropwise manner; the solutions were left to incubate overnight at $4{ }^{\circ} \mathrm{C}$. The following day, the slices were incubated with horseradish peroxidase (HRP)-labelled goat anti-rabbit/mouse secondary antibodies and stained using 3,3-diaminobenzidine (UltraSensitive ${ }^{\mathrm{TM}}$ SP Mouse/Rabbit IHC Kit, Fuzhou Maixin Biotech Co. Ltd., Fuzhou, China). Nuclei were stained blue using haematoxylin. The sections were then dehydrated, cleared by xylene, and mounted.

Samples were independently observed, scored, and evaluated by two pathologists who were blinded to the clinical information of the patients. If discordant scoring 
results were obtained, a third pathologist would assess the sample for the final decision. The samples were classified as positive when presenting with a brownish-yellow, or brown colour in the cell cytoplasm and/or membrane. If the proportion of positive cells was less than $5 \%$, it was scored as 0 points, $5-25 \%$ was scored as 1 point, $26-50 \%$ was 2 points, $51-75 \%$ was 3 points, and more than $75 \%$ was scored as 4 points. According to the colour intensity, samples were further scored with 3 points for brown, 2 points for brownish-yellow, 1 point for light yellow, and 0 points for no staining. To reach a final score, the two classifications were multiplied: $0-2$ points was recorded as negative expression (-), 3-4 points as weakly positive expression (+), 5-8 points as moderately positive expression $(++)$, and $9-12$ points as strongly positive expression $(+++)$.

\section{Cellular immunofluorescence}

Cells were seeded on a microscope slide and washed with phosphate-buffered saline (PBS) after they had adhered to the glass. Goat serum blocking solution was added, followed by the anti-YWHAE (1:100, sc-23957, Santa Cruz Biotechnology, Santa Cruz, CA) and anti-HE4 (1:200, DF8160, Affinity Biosciences, Cincinnati, OH) primary antibody mix. A secondary antibody mixture containing tetramethylrhodamine-labelled goat anti-rabbit IgG (SA00007-2, Proteintech Group Inc., Wuhan, China) and fluorescein isothiocyanate-labelled goat anti-mouse IgG (SA00003-1, Proteintech Group Inc., Wuhan, China) was dropped onto the slide, which was then incubated for $2 \mathrm{~h}$ in the dark. Nuclei were stained with 4',6-diamidino2-phenylindole (4083S; Cell Signaling Technology Inc., Danvers, MA), and an anti-quenching agent was added dropwise onto the slide immediately before viewing the slices on a confocal microscope.

\section{Establishment of stable cell lines overexpressing YWHAE and transient YWHAE knockdown cell lines}

The ovarian cancer cell lines, CAOV3 and ES2 (Shanghai Institute of Biochemistry and Cell Biology, Chinese Academy of Sciences, Shanghai, China), were cultured in RPMI 1640 Medium (Biological Industries, Beit-Haemek, Israel) supplemented with $10 \%$ foetal bovine serum (Biological Industries, Beit-Haemek, Israel). When the cells reached $80-90 \%$ confluence, the medium was discarded, the cells were washed with PBS, trypsinized, and split to continue the culture. $Y W H A E$-siRNA (5'-GAAGCAGGU UAGCGUUGAATT- $3^{\prime}$ and $3^{\prime}$-UUCAACGCUAACCUG CUUCTT-5'), Mock-YWHAE-siRNA (5'-GAAGCAGGU UAGCGUUAGATTUU- $3^{\prime}$ and $3^{\prime}$-UUCTTCGCUAAC CCTCUUCTT-5') (Additional file 1), HE4-siRNA (5'AGGUGAACAUUAACUUUCCTT-3' and $3^{\prime}$-GGAAAG UUAAUGUUCACCUTT-5' ${ }^{\prime}$ ), and Mock-HE4-siRNA
(5'-AGGUGAACACCAACTTUCCTT-3' and $3^{\prime}$-GGA AAGCCAAUGUUTCCCUTT-5') working solutions were prepared according to the manufacturer's instructions (GenePharma, Suzhou, China). CAOV3 and ES2 cells were seeded into 6-well plates with serum-free medium on the day of transfection. YWHAE-siRNA and HE4-siRNA were transfected into these cell lines using Lipofectamine 3000 (Invitrogen, Waltham, MA, USA) according to the manufacturer's instructions.

A lentivirus-mediated YWHAE overexpression vector was used to transduce OVCAR3 and A2780 cell lines, which have relatively low YWHAE expression. To determine the multiplicity of infection of the YWHAEexpressing lentivirus, $500 \mu \mathrm{L}$ of complete medium was added to a 24-well plate, in addition to lentivirus supernatant and a corresponding volume of polybrene to promote transduction. Puromycin at $50 \%$ lethal concentration was used to select the transduced cells.

\section{Western blotting}

Total protein samples were separated using sodium dodecyl sulphate polyacrylamide gel electrophoresis. Briefly, 5-10 $\mu \mathrm{L}$ of each protein sample was electrophoresed at $80-120 \mathrm{~V}$ under a constant electrical current for 40-100 min. The proteins were then transferred onto a polyvinylidene fluoride membrane (Millipore, Burlington, MA), and blocked with a $5 \%$ milk/bovine serum albumin solution for $2 \mathrm{~h}$ at $37{ }^{\circ} \mathrm{C}$. The membrane was incubated overnight at $4{ }^{\circ} \mathrm{C}$ with primary antibodies (YWHAE, 1:1000, ab92311, Abcam, Cambridge, UK; HE4, 1:1500, DF8160, Affinity Biosciences, Cincinnati, OH; cyclin D1, 1:1000, 2978S, Cell Signaling Technology Inc., Danvers, MA; Ki-67, 1:1000, 9449S, Cell Signaling Technology Inc., Danvers, MA; Bax, 1:1000, 5023S, Cell Signaling Technology Inc., Danvers, MA; Bcl-2, 1:1000, 12789-1-AP, Proteintech Group Inc., Wuhan, China; MMP2, 1:1000, 10373-2-AP, Proteintech Group Inc., Wuhan, China; MMP9, 1:1000, 10375-2-AP, Proteintech Group Inc., Wuhan, China; E-cadherin, 1:1000, 20874-1-AP, Proteintech Group Inc., Wuhan, China; N-cadherin, 1:1000, 4061S, Cell Signaling Technology Inc., Danvers, MA; Vimentin, 1:3000, 10366-1-AP, Proteintech Group Inc., Wuhan, China; PI3K, 1:1000, 4292S, Cell Signaling Technology Inc., Danvers, MA; p-PI3K, 1:1000, 4228S, Cell Signaling Technology Inc., Danvers, MA; AKT, 1:1000, 4691S, Cell Signaling Technology Inc., Danvers, MA; p-AKT, 1:1000, 4060S, Cell Signaling Technology, Beverly, MA; m-TOR, 1:1000, 2972S, Cell Signaling Technology Inc., Danvers, MA; p-m-TOR, 1:1000, 2971S, Cell Signaling Technology Inc., Danvers, MA; MEK, 1:1000, sc-81504, Santa Cruz Biotechnology, Santa Cruz, CA; p-MEK, 1:1000, 2338S, Cell Signaling Technology Inc., Danvers, MA; ERK, 1:1000, 9102S, Cell 
Signaling Technology Inc., Danvers, MA; and p-ERK, 1:1000, 9101S, Cell Signaling Technology Inc., Danvers, MA). After washing the membrane with PBS-Tween, the membrane was incubated with HRP-labelled goat antirabbit (1:3000, ZB-2301, ZSGB-BIO, Beijing, China) or goat anti-mouse (1:3000, ZB-2305, ZSGB-BIO, Beijing, China) secondary antibodies for $1 \mathrm{~h}$ at $37{ }^{\circ} \mathrm{C}$, and then washed again. Finally, western chemiluminescent HRP Substrate (Thermo Fisher Scientific, Waltham, MA) was added dropwise onto the membrane, and luminescent signals were detected on a luminometer at different exposure times.

\section{Immunoprecipitation}

Cells in the exponential growth phase were collected and washed. Ice-cold lysis buffer was added, and the cell suspension was sonicated. The supernatant was collected and the total protein concentration was determined. Samples $(500 \mu \mathrm{g})$ were then incubated with $2 \mu \mathrm{g}$ of YWHAE (1:100, sc-23957, Santa Cruz Biotechnology, Santa Cruz, CA) or HE4 (1:1500, ab200828, Abcam, Cambridge, UK) primary antibodies. An IgG antibody (5145S, Cell Signaling Technology Inc., Danvers, MA) of the same species as the primary antibodies was used as a negative control. Following mixing, the samples were rotated slowly overnight at $4{ }^{\circ} \mathrm{C}$. Subsequently, Protein A/G Plus-Agarose Beads (sc-2003, Santa Cruz Biotechnology, Santa Cruz, CA) were added to each tube and incubated for $6 \mathrm{~h}$. Bound proteins were collected via centrifugation, $(2 \times)$ loading buffer was added, and the samples were boiled to denature the proteins.

\section{Invasion test}

A transwell insert was placed into a 24-well plate. Matrigel (1:7.5 dilution, 356234, BD Biosciences, Franklin Lakes, NJ) and serum-free cell suspensions $(4 \times 10$ cells $)$ were added to the upper insert of the chamber, whereas the lower chamber contained complete medium (with $10 \%$ serum). After culturing for $48-72 \mathrm{~h}$, the cells in the lower chamber were collected, fixed, and stained. Residual cells left on plate were observed under a microscope. The experiment was repeated thrice.

\section{Scratch test}

Cells were seeded in a 6-well culture plate and maintained at $37{ }^{\circ} \mathrm{C}$ in an atmosphere of $5 \% \mathrm{CO}_{2}$. When the cells were at $90 \%$ confluence, the monolayer was scratched with a $100 \mu \mathrm{L}$ pipette tip. Cells were cultured in serum-free medium for $24 \mathrm{~h}$, and then washed with PBS and imaged to monitor the healing of the scratch. The experiment was repeated thrice.

\section{Cell viability assay}

A total of $2 \times 10^{3}$ cells/well were seeded in to a 96-well culture plate and maintained at $37{ }^{\circ} \mathrm{C}$ in an atmosphere of $5 \% \mathrm{CO}_{2}$. After the cells had adhered, $20 \mu \mathrm{L}$ of sterile MTT (M8180,Solarbio, Beijing, China) working solution was added to each well, mixed well, and the cells incubated at $37^{\circ} \mathrm{C}$ for $4 \mathrm{~h}$. Following this, the medium was aspirated and $150 \mu \mathrm{L}$ of DMSO (D8370, Beijing Solarbio Science \& Technology Co Ltd., Beijing, China) was added to each well. The absorbance of each well was measured using a microplate reader after shaking for $5 \mathrm{~min}$. The experiment was repeated thrice.

\section{Cell cycle analysis}

Cells in the log phase of replication were collected and washed, and pre-cooled ethanol was slowly added to fix the cells for later use. Prior to analysis, a total of $500 \mu \mathrm{L}$ PI/RNase A (KGA512, Nanjing KeyGen Biotech Co Ltd., Nanjing, China) staining solution was added to the cell suspension, and the cells were incubated in the dark for $20 \mathrm{~min}$, according to the manufacturer's instructions. The cells were analysed via flow cytometry. The experiment was repeated thrice.

\section{Apoptosis assessment}

The annexin V/PI double staining method was used to evaluate the impact of YWHAE overexpression on ovarian cancer cells. The cells were trypsinized without EDTA, centrifuged, and the supernatant was discarded. Cells were then slowly resuspended in $500 \mu \mathrm{L}$ of Binding Buffer and incubated with $5 \mu \mathrm{L}$ of annexin V-APC (550474, BD Biosciences, Franklin Lakes, NJ)/FITC (KGA107, Nanjing KeyGen Biotech Co Ltd., Nanjing, China) and PI in the dark at $37{ }^{\circ} \mathrm{C}$ for $15 \mathrm{~min}$, according to the manufacturer's instructions. Apoptosis was evaluated via flow cytometry.

\section{In vivo xenograft model of ovarian cancer}

Twenty female nude mice (Huafukang Biosciences, Beijing, China) were randomly divided into two groups and injected with either OVCAR3-YWHAE-MOCK or OVCAR3-YWHAE-H ovarian cancer cells. Approximately $100 \mu \mathrm{L}$ of cell suspension containing $1 \times 10^{7}$ cells was injected subcutaneously into the armpit of the right forelimb of each mouse. Tumour progression and overall health status of the mice were observed every 3 days, and the diameter of the tumour and the weight of the mice were measured. The tumour volume was calculated using the following formula: $V=\left(a \times b^{2}\right) / 2$, where (a) represents the largest diameter, and (b) the shortest diameter. On day 7 post-injection, newly formed tumours were detectable; on day 21 , the largest tumour was nearly 
$1 \mathrm{~cm}$ in diameter, and the mice had started to show poor health symptoms. The tumour samples were then fixed in $4 \%$ paraformaldehyde and embedded in paraffin. Continuous $5 \mu \mathrm{m}$-thick sections were cut and analysed using either haematoxylin and eosin or immunohistochemical staining. This animal study was approved by the Institutional Animal Research Committee of China Medical University.

\section{Signalling pathway inhibition}

YWHAE-overexpressed and mock-transduced cells were cultured in the presence of $25 \mu \mathrm{mol} / \mathrm{L}$ of the PI3K inhibitor (GDC-0941, Selleck Chemicals, Houston, TX) or $20 \mu \mathrm{mol} / \mathrm{L}$ of a selective inhibitor of mitogen-activated protein kinase 1 and 2 (MAPK1/2) (PD98059, Selleck Chemicals, Houston, TX). DMSO (0.1\%) served as the negative control.

\section{Bioinformation analysis}

The YWHAE co-expression gene set was downloaded from cBioPortal (www.cbioportal.org), and the top 300 genes were annotated according to the Gene Ontology (GO) (BP, biological process; CC, cellular component; and MF, molecular function) and the Kyoto Encyclopaedia of Genes and Genomes (KEGG) pathway analysis databases, using the tools available in DAVID (https:// david.ncifcrf.gov/).

The Oncomine database (http://www.oncomine.org), which is currently the world's largest oncogene chip database integrated with a data mining platform, was used to analyse the mRNA expression level of YWHAE in the different cancer cell types.

The interaction network among the top 100 genes was constructed using STRING (www.string-db.org) and Cytoscape (www.cytoscape.org). The protein-protein interaction (PPI) network was constructed using the STRING (http://string.embl.de/) online tool. The visualization plot was generated using Cytoscape software with a confidence score of $\geq 0.1$ defined as the cut-off. The core modules of the PPI network were screened using MCODE with the following parameters: degree threshold $=2$, node threshold $=0.2$, kcore $=2$, and maximum depth $=100$.

\section{Statistical analysis}

Statistical differences between two groups were evaluated using a Student's $t$ test, and one-way analysis of variance
(ANOVA) was used for comparison of more than two groups. The data were counted using the $\chi^{2}$ and Fisher's exact probability tests. $P<0.05$ was considered statistically significant.

\section{Results}

YWHAE and HE4 are interacting proteins in ovarian cancer The levels of YWHAE and HE4 were evaluated in CAOV3 and ES2 ovarian cancer cell lines using cellular immunohistochemistry, revealing that both proteins were expressed in these lines (Fig. 1a), co-localising in the cytoplasm and cell membranes (Fig. 1b). Co-immunoprecipitation of YWHAE and HE4 in these cell lines further demonstrated that they are interacting proteins (Fig. 1c). Additionally, siRNA-mediated knockdown of YWHAE led to reduced levels of HE4 in both CAOV3 and ES2 transiently transfected cells, whereas HE4 knockdown had no significant effect on YWHAE levels. These results suggest that YWHAE works upstream of HE4 (Fig. 1d,e), and can regulate HE4 expression.

DAVID analysis of the top 300 genes identified on the GO (BP/CC/MF) and KEGG databases showed an enrichment of $36 \mathrm{BP}$, which included RNA scattering, Wnt signalling, and cell adhesion, $23 \mathrm{CC}$, and 13 MF (all $P<0.05$ ). In addition, two KEGG signalling pathways were identified (all $P<0.05$ ), which included spliceosome and RNA transport. A bubble diagram of the top five GO (BP/CC/MF) and KEGG pathways showed that YWHAE may interact with PAFAH1B1, EIF5A, PITPNA, TIMM22, PFN1, and WFDC2 (which is another name of HE4) (Fig. 1f).

\section{Expression and correlation analysis of YWHAE and HE4 in each group of ovarian tissues YWHAE expression in ovarian tissues}

Consistent with the results from cell line studies, analysis of primary tissue samples showed that YWHAE was mainly located in the cytoplasm and cell membrane. Approximately $96.19 \%$ (101/105) of the malignant samples were positive for YWHAE with a strongly positive rate of $70.48 \%(74 / 105)$, whereas the borderline group had a YWHAE-positive rate of $41.67 \%(10 / 24)$, and a strongly positive rate of $16.67 \%(4 / 24)$. Benign and normal ovarian tissue samples had a positive rate of $16.67 \%(3 / 18)$ and $6.25 \%(1 / 16)$, and a strongly positive rate of $11.11 \%(2 / 18)$ and $0.00 \%(0 / 16)$, respectively. Comparison between the different groups revealed that YWHAE-positive expression was significantly higher in the malignant group $(P<0.05)$; however, the borderline group also had a higher YWHAE-positive rate than that of the benign and normal groups $(P<0.05)$. The expression rate of YWHAE in the benign group was higher 


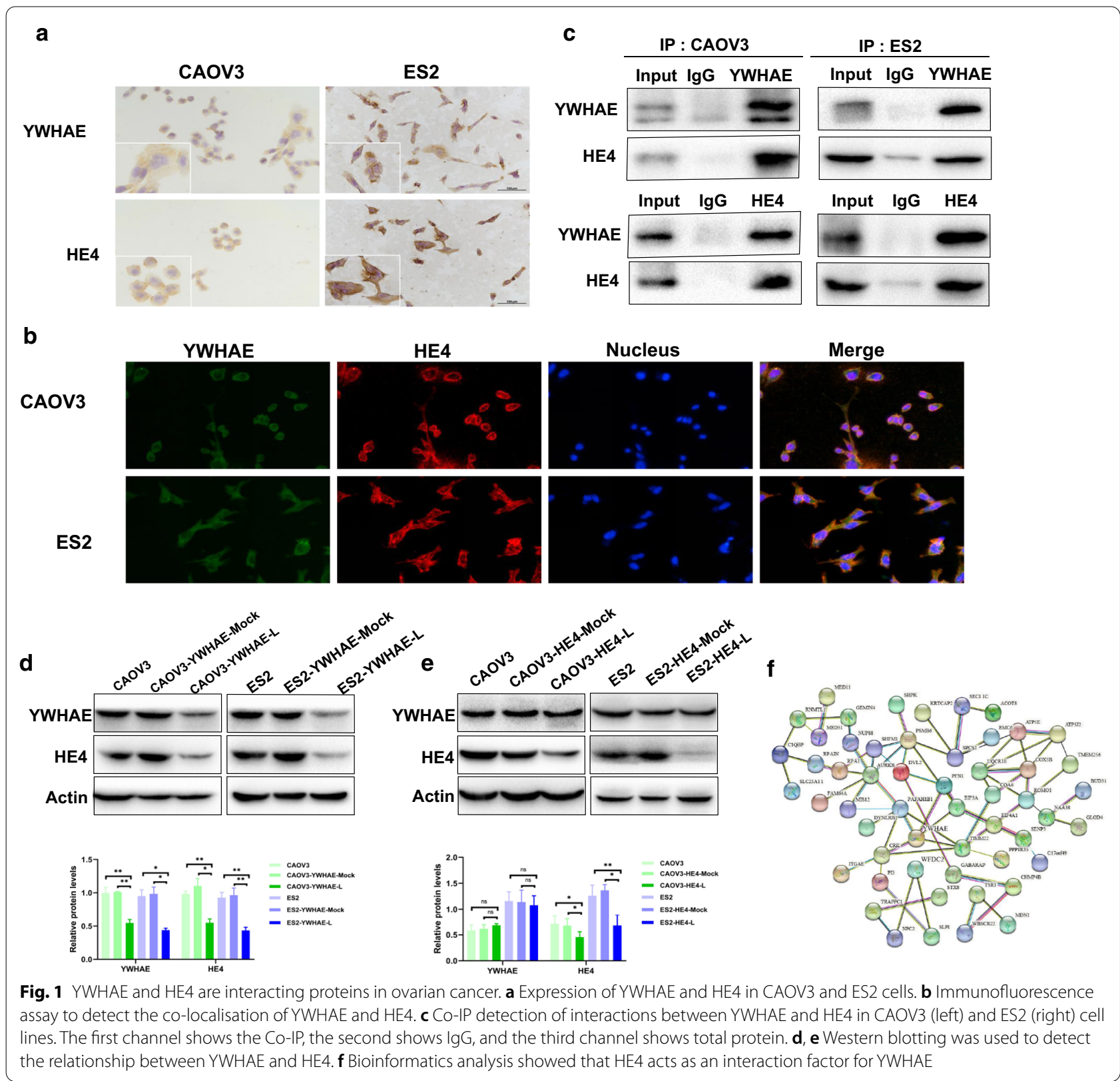

Table 1 Expression of YWHAE in different ovarian tissues

\begin{tabular}{|c|c|c|c|c|c|c|c|}
\hline \multirow[t]{2}{*}{ Groups } & \multirow[t]{2}{*}{ Cases } & \multicolumn{2}{|c|}{ Low } & \multicolumn{2}{|c|}{ High } & \multirow[t]{2}{*}{ Positive rate (\%) } & \multirow{2}{*}{$\begin{array}{l}\text { High } \\
\text { expression } \\
\text { rate (\%) }\end{array}$} \\
\hline & & - & + & ++ & +++ & & \\
\hline Malignant & 105 & 4 & 27 & 36 & 38 & 96.19 & 70.48 \\
\hline Borderline & 24 & 14 & 6 & 2 & 2 & 41.67 & 16.67 \\
\hline Benign & 18 & 15 & 1 & 2 & 0 & 16.67 & 11.11 \\
\hline Normal & 16 & 15 & 1 & 0 & 0 & 6.25 & 0.00 \\
\hline
\end{tabular}




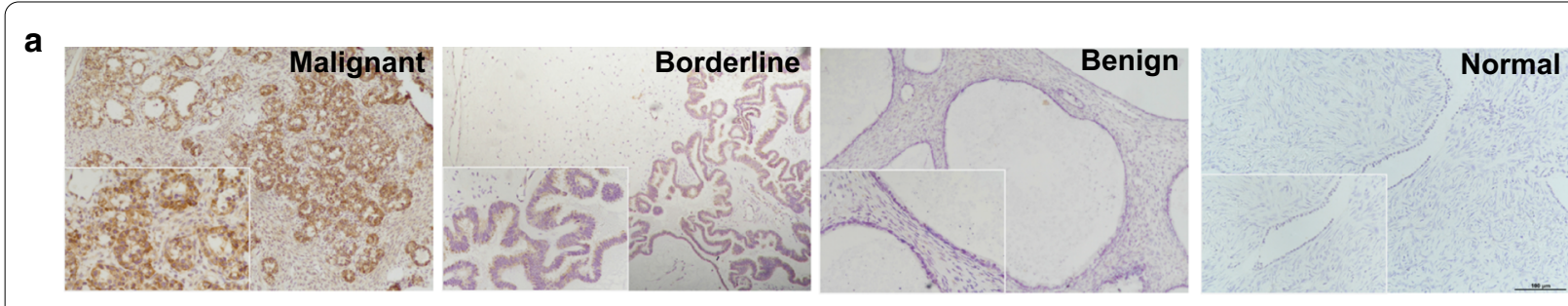

b
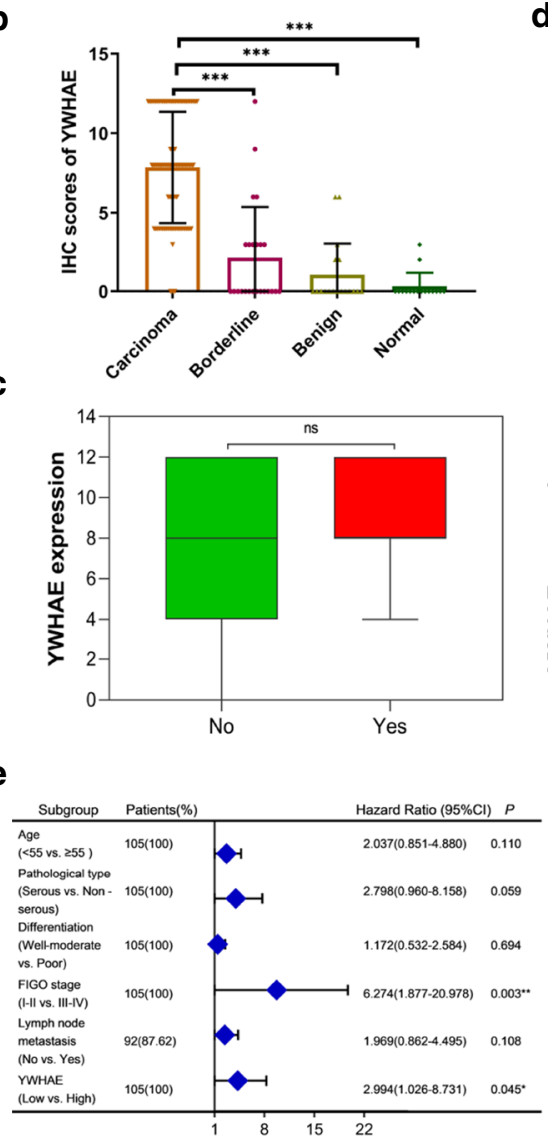

g

Disease Summary for YWHAE

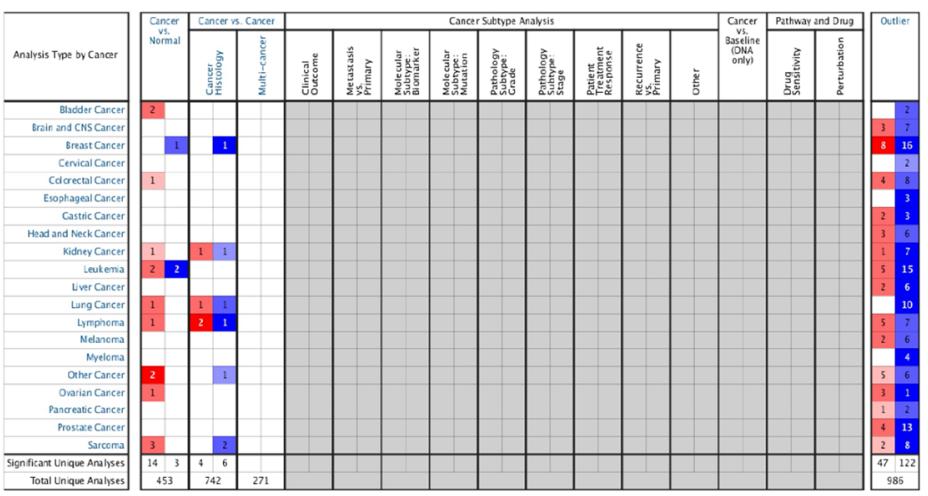

d
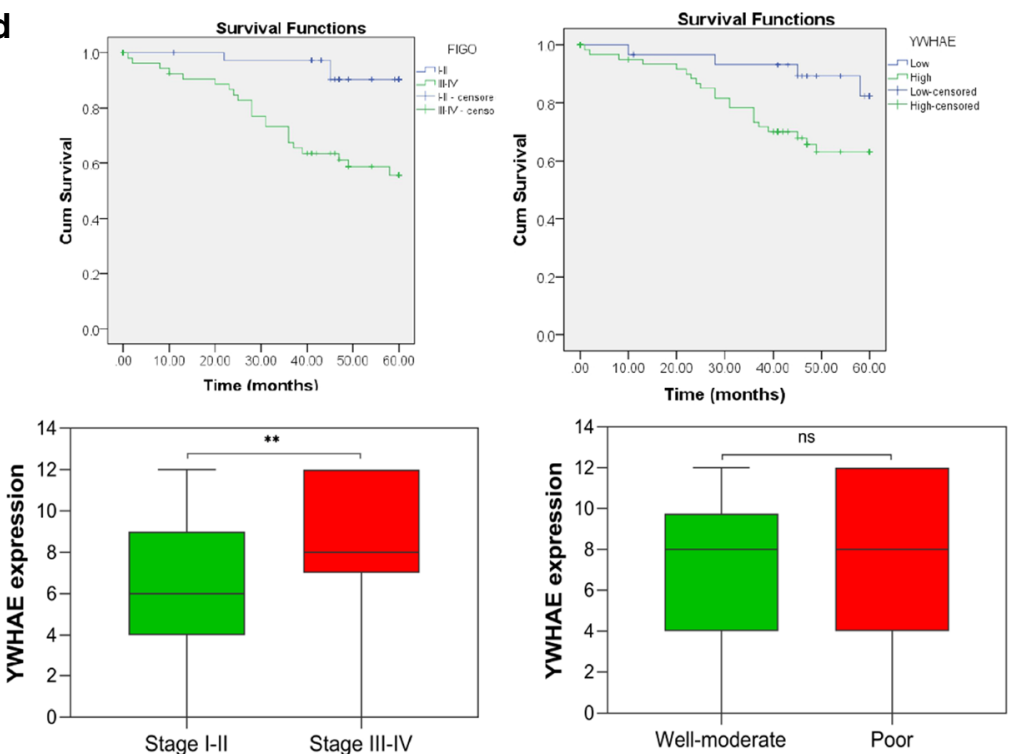

f

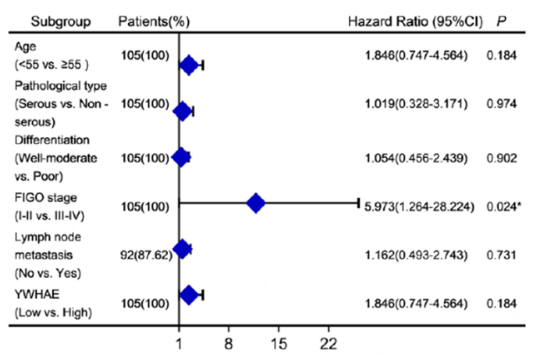

h

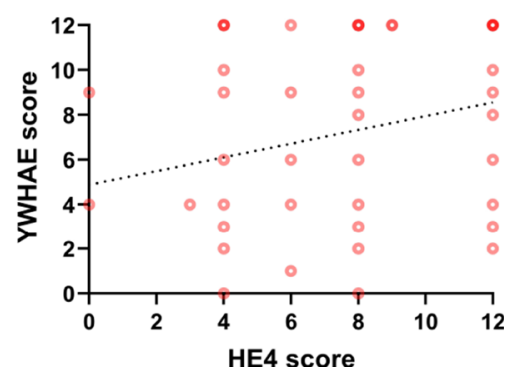

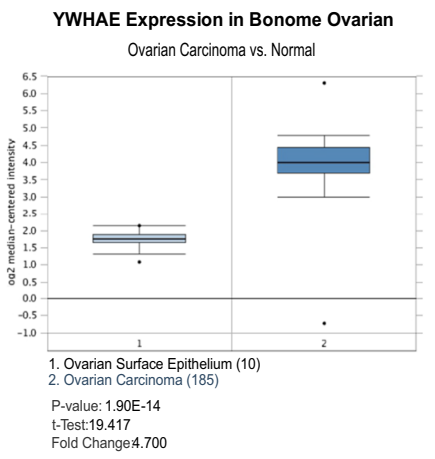

Fig. 2 YWHAE expression in clinical specimens. a The expression of YWHAE and HE4 in the same position in malignant, borderline, benign, and normal tissues. b YWHAE scores in different ovarian tissues. c Comparison of YWHAE expression between groups with and without lymph node metastasis, FIGO stage I-II vs. III-IV, and groups with well-moderate or poor differentiation. $\mathbf{d}$ The influence of FIGO stage and YWHAE expression on the survival and prognosis of ovarian cancer patients. e Univariate and multivariate Cox analyses of different clinicopathological parameters of ovarian cancer. $\mathbf{f}$ Linear correlation analysis between YWHAE and HE4 expression in ovarian cancer; $\mathbf{g}, \mathbf{h}$ YWHAE expression in Oncomine database 
than in the normal group, however the difference was not statistically significant $(P>0.05)$ (Table 1 , Fig. 2a, b).

\section{Relationship between YWHAE expression and the clinicopathological parameters of ovarian cancer}

To compare clinicopathological parameters with the expression of YWHAE in ovarian tissue, we reviewed clinical information of the 105 patients with primary malignant epithelial ovarian tumours. Analysis of the pathological data showed that a strongly positive YWHAE expression rate was significantly higher in FIGO stage III-IV ovarian epithelial malignancies than in the early-stage group $(80.33 \%$ versus $56.82 \%, P<0.01)$. No statistical differences were observed in any other clinicopathological parameters (Table 2, Fig. 2c).

\section{Relationship between YWHAE expression and the survival of patients with ovarian cancer}

A follow-up of these patients further revealed that only four deaths occurred in the low YWHAE expression group $(n=31)$, while 21 deaths were recorded in the high YWHAE expression group $(n=74)$. Kaplan-Meier survival analysis showed that the survival rate among patients with high YWHAE expression was significantly lower compared with those in the low YWHAE expression group. This result followed the same trend when comparing patients with early or late FIGO staging $(P<0.05)$ (Fig. $2 \mathrm{~d})$. Univariate and multivariate Cox regression analysis of YWHAE expression with age, pathological type, degree of differentiation, FIGO stage, and lymph node metastasis, demonstrated that YWHAE expression and FIGO staging are risk factors for the prognosis of epithelial ovarian malignancies (Fig. 2e).

\section{YWHAE and HE4 expressions are related in ovarian cancer tissues}

Next, the co-expression of YWHAE and HE4 was evaluated in 80 cases of ovarian cancer. A total of 0 , three, nine, and 68 cases were YWHAE-/HE4-, YWHAE-/HE4+, YWHAE+/HE4-, and YWHAE+/HE4+, respectively. Spearman's correlation analysis confirmed that YWHAE and HE4 expression is positively correlated in ovarian cancer (correlation coefficient Rs $=0.277, P=0.013$ ) (Table 3, Fig. 2f). Linear regression analysis showed that YWHAE and HE4 can influence the expression of each

Table 3 Correlation between YWHAE and HE4 in ovarian cancer

\begin{tabular}{llrc}
\hline YWHAE & \multicolumn{2}{c}{ HE4 } & Cases \\
\cline { 2 - 3 } & - & + & \\
\hline- & 0 & 3 & 3 \\
+ & 9 & 68 & 77 \\
Cases & 9 & 71 & 80 \\
\hline
\end{tabular}

Sperman correlation coefficient $\mathrm{Rs}=0.277, P=0.013$

Table 2 Relationships between the expression of YWHAE and clinicopathological parameters

\begin{tabular}{|c|c|c|c|c|c|c|c|c|c|}
\hline \multirow[t]{2}{*}{ Groups } & \multirow[t]{2}{*}{ Cases } & \multicolumn{2}{|c|}{ Low } & \multicolumn{2}{|l|}{ High } & \multirow[t]{2}{*}{ Positive rate (\%) } & \multirow[t]{2}{*}{$P$-value } & \multirow{2}{*}{$\begin{array}{l}\text { High expression } \\
\text { rate }(\%)\end{array}$} & \multirow[t]{2}{*}{$P$-value } \\
\hline & & $(-)$ & $(+)$ & $(++)$ & $(+++)$ & & & & \\
\hline \multicolumn{10}{|l|}{ Age at diagnosis } \\
\hline$<55$ & 54 & 4 & 13 & 18 & 19 & 92.59 & $P=0.066$ & 68.52 & $P=0.651$ \\
\hline$\geq 55$ & 51 & 0 & 14 & 18 & 19 & 100.00 & & 72.55 & \\
\hline \multicolumn{10}{|l|}{ Pathological type } \\
\hline Serous & 71 & 2 & 14 & 28 & 27 & 97.18 & $P=0.222$ & 77.46 & $P=0.073$ \\
\hline Mucinous & 7 & 1 & 3 & 2 & 1 & 85.71 & & 42.86 & \\
\hline Endometrioid & 19 & 1 & 6 & 4 & 8 & 94.73 & & 63.16 & \\
\hline Clear cell carcinoma & 8 & 0 & 4 & 2 & 2 & 100.00 & & 50.00 & \\
\hline \multicolumn{10}{|l|}{ FIGO stage } \\
\hline$|-| \mid$ & 44 & 3 & 16 & 12 & 13 & 93.18 & $P=0.307$ & 56.82 & $P=0.009$ \\
\hline III-IV & 61 & 1 & 11 & 24 & 25 & 98.36 & & 80.33 & \\
\hline \multicolumn{10}{|l|}{ Differentiation } \\
\hline Well and moderate & 51 & 3 & 13 & 12 & 23 & 94.12 & $P=0.354$ & 68.63 & $P=0.687$ \\
\hline Poor & 54 & 1 & 14 & 24 & 15 & 98.15 & & 72.22 & \\
\hline \multicolumn{10}{|l|}{ Lymphatic metastasis } \\
\hline No & 64 & 4 & 18 & 22 & 20 & 93.75 & $P=0.310$ & 65.63 & $P=0.214$ \\
\hline Yes & 28 & 0 & 6 & 10 & 12 & 100.00 & & 78.57 & \\
\hline Unknown ${ }^{a}$ & 13 & 0 & 3 & 4 & 6 & 100.00 & & 76.92 & \\
\hline
\end{tabular}

a 13 patients without lymphadenectomy 
Table 4 Linear regression analysis of YWHAE and HE4

\begin{tabular}{|c|c|c|c|c|c|c|c|c|}
\hline & \multicolumn{4}{|c|}{ YWHAE score } & \multicolumn{4}{|c|}{ HE4 score } \\
\hline & \multicolumn{2}{|c|}{ Univariate } & \multicolumn{2}{|c|}{ Multivariate } & \multicolumn{2}{|c|}{ Univariate } & \multicolumn{2}{|c|}{ Multivariate } \\
\hline & $\beta$ & $P$ & $\beta$ & $P$ & $\beta$ & $P$ & $\beta$ & $P$ \\
\hline HE4 score & 0.307 & 0.009 & 0.307 & $0.009^{\mathrm{a}}$ & & & & \\
\hline YWHAE score & & & & & 0.272 & 0.009 & 0.235 & $0.025^{b}$ \\
\hline Age at diagnosis & -0.238 & 0.772 & & & 0.137 & 0.859 & & \\
\hline FIGO stage & 1.429 & 0.087 & & & 1.772 & 0.023 & 1.436 & 0.062 \\
\hline Differentiation & -0.203 & 0.809 & & & 0.139 & 0.861 & & \\
\hline Lymphatic metastasis & -0.017 & 0.986 & & & 1.383 & 0.118 & & \\
\hline
\end{tabular}

${ }^{a}$ Represents multi-factor linear regression analysis, with YWHAE score as a variable, HE4 score as an argument;

${ }^{\mathrm{b}}$ Represents multi-factor linear regression analysis, with HE4 score as a variable, with YWHAE score and FIGO stage as arguments

other $(P<0.05)$, and that the late FIGO stages are important factors affecting HE4 expression (Table 4).

\section{YWHAE expression correlates with ovarian cancer tissues}

The Oncomine database showed that YWHAE was highly expressed in the cancer group compared to the normal tissue group. For ovarian cancer, YWHAE expression was significant in 185 ovarian carcinoma tissues compared with 10 ovarian surface epithelium tissues (Fig. 2g, h).

YWHAE promotes ovarian cancer cell invasion, migration, and epithelial-mesenchymal transition potential

Analysis of YWHAE expression in several ovarian cancer cell lines revealed higher levels in CAOV3 and ES2 than in OVCAR3 and A2780 lines. Based on these results, CAOV3 and ES2 cells were used to establish YWHAE knockdown cell lines, whereas OVCAR3 and A2780 cells were used to establish stable YWHAE overexpression cell lines (Fig. 3a-f).

The effect on ovarian cancer cell invasion and migration upon transient knockdown, or stable overexpression of YWHAE was next evaluated by transwell, and scratch experiments. Overall, the data revealed that both OVCAR3 and A2780 cells overexpressing YWHAE had significantly enhanced invasion and migration capacity in comparison with mock-transduced, and untransduced cells. In contrast, CAOV3 and ES2 cells reducing YWHAE showed weaker invasion and migration abilities compared with control cells (all $P<0.05)$ (Fig. $3 g-n)$. To further explore the impact of YWHAE on the behaviour of ovarian cancer cells, the expression of epithelial and mesenchymal markers was evaluated in these cells by western blot. YWHAE-overexpression was found to be associated with higher levels of $\mathrm{N}$-cadherin, vimentin, MMP2, and MMP9 (cell mesenchymal markers), while the epithelial marker E-cadherin was reduced when compared with cells in the control groups. The opposite trend was observed when YWHAE was knocked-down (both $P<0.05)$, with higher levels of E-cadherin and reduced levels of N-cadherin, vimentin, MMP2, and MMP9 (Fig. 4a-d). Altogether, these results demonstrate that YWHAE promotes invasion, migration, and epithelialmesenchymal transition of epithelial ovarian cancer cells.

\section{YWHAE promotes ovarian cancer by inducing cell proliferation and cell cycle progression and inhibiting apoptosis}

Additional assessment of the OVCAR3 and A2780 YWHAE overexpression cell lines further showed that most cells were in G2/M phase, suggesting that they were actively proliferating compared with mock-transduced and untransduced cells (all $P<0.05$ ). Furthermore, these cells showed higher expression of Ki67, cyclin D1, and Bcl-2, and reduced levels of Bax (all $P<0.05$ ). CAOV3 and ES2 knockdown YWHAE lines showed the opposite results, with a significantly low proportion of cells in the G2/M phase, reduced levels of proliferation markers, and increased levels of the Bax apoptosis marker. Furthermore, flow cytometry revealed that compared with the control group, apoptosis of OVCAR3 and A2780 cells was significantly reduced following YWHAE overexpression $(P<0.05)$. In contrast, apoptosis was significantly increased in OVCAR3 and A2780 cells when YWHAE expression was inhibited, compared with the control group $(P<0.05)$ (Figs. $4 \mathrm{e}-\mathrm{h}, 5)$. Taken together, these results demonstrate that YWHAE expression can enhance the proliferation of ovarian cancer cells while also promoting cell cycle progression and inhibiting cellular apoptosis.

\section{Effect of YWHAE on the in vivo tumorigenesis of ovarian cancer cells}

To explore the effect of YWHAE on the tumorigenic ability of ovarian cancer cells, OVCAR3 cells overexpressing 


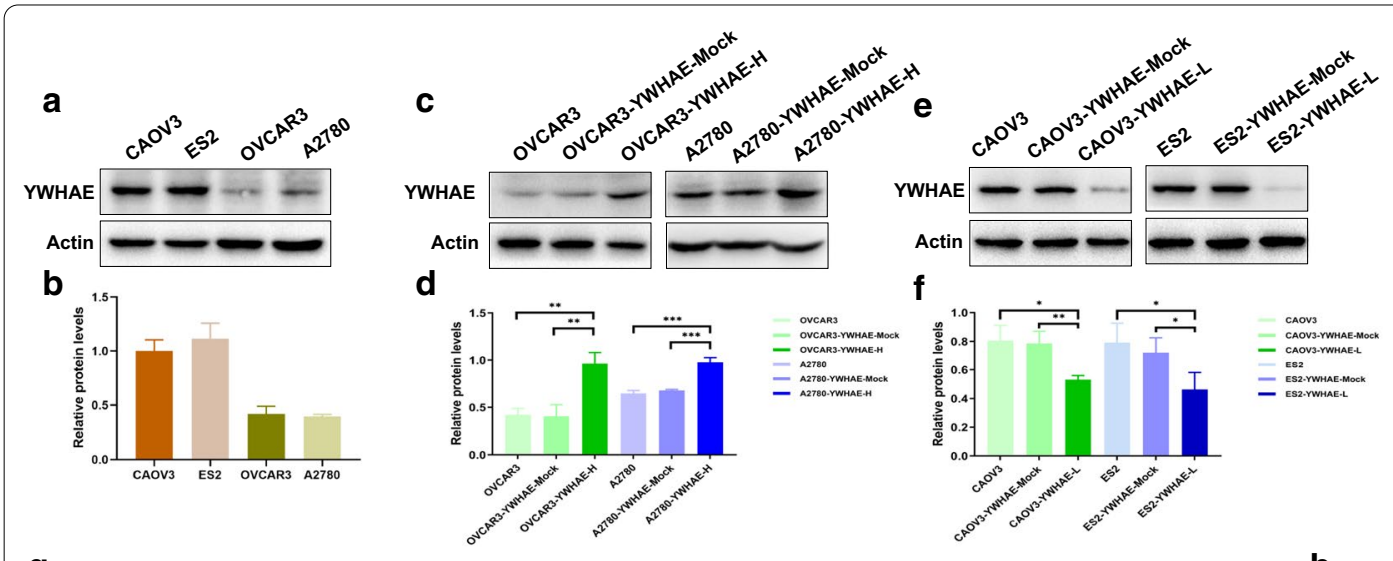

g

OVCAR3 OVCAR3-YWHAE-Mock OVCAR3-YWHAE-H

A2780

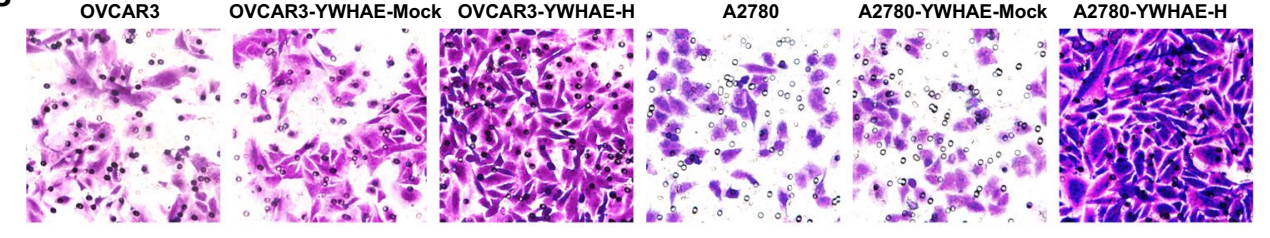

h

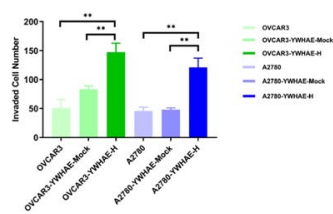

i CAOV3 CAOV3-YWHAE-Mock CAOV3-YWHAE-L

ES2 ES2-YWHAE-Mock ES2-YWHAE-H
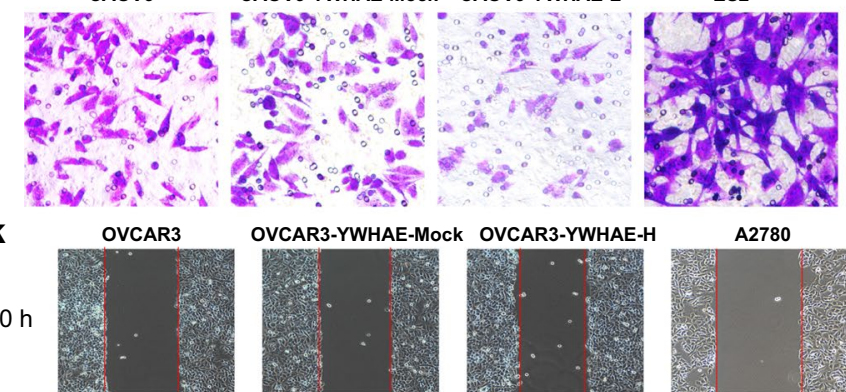

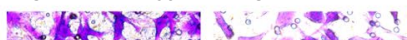

j
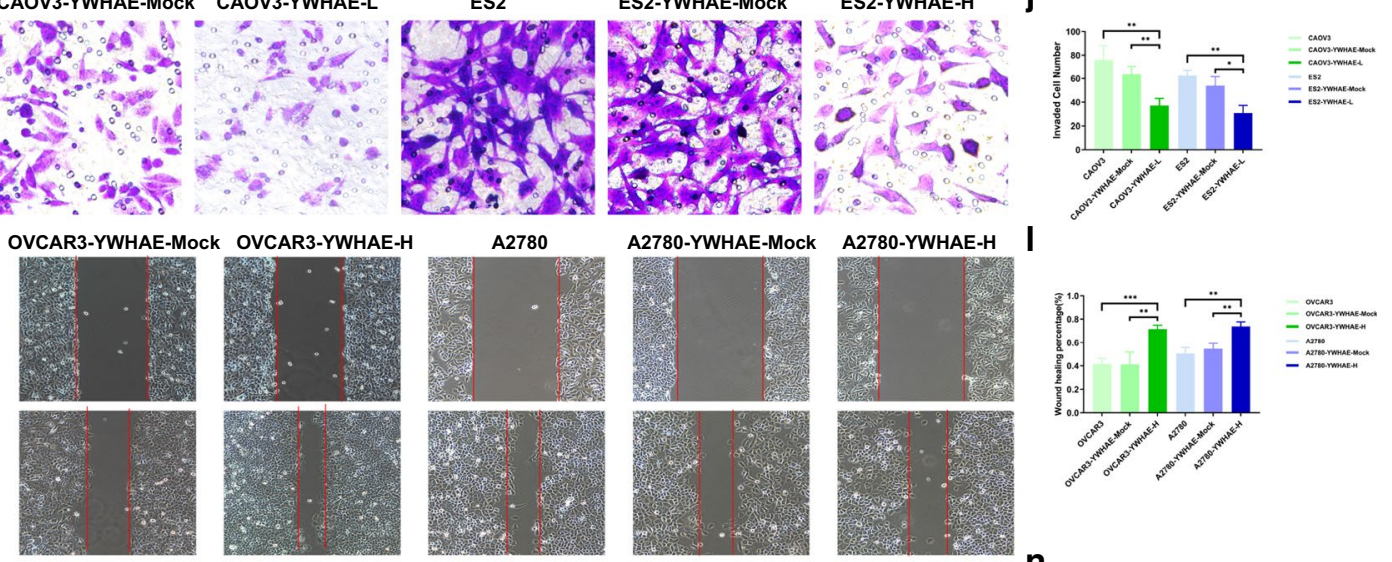

CAOV3-YWHAE-L

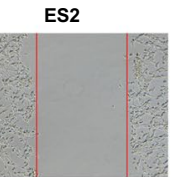

ES2-YWHAE-Mock
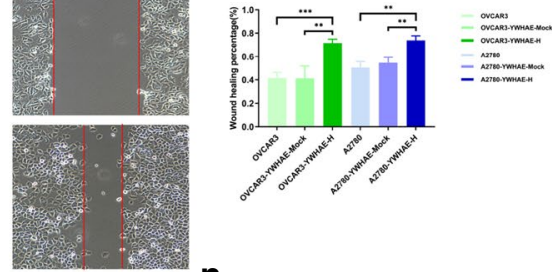

m

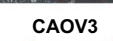

CAOV3-YWHAE-Mock
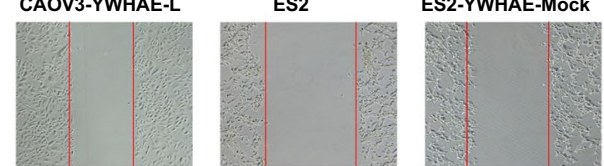

ES2-YWHAE-H
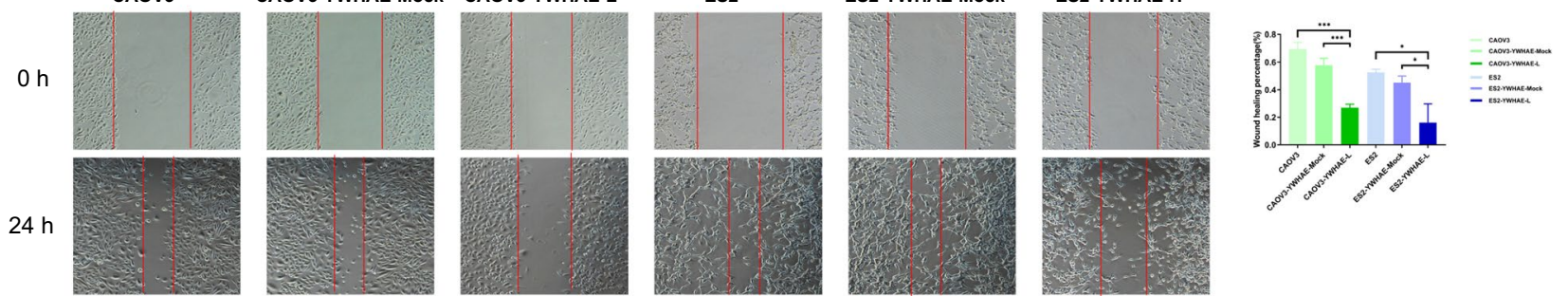

Fig. 3 YWHAE affects cell invasion, migration, and epithelial-mesenchymal transition in ovarian cancer. $\mathbf{a}, \mathbf{b}$ Y YWHAE expression in ovarian cancer cell lines. $\mathbf{c}$, d Western blot analysis of YWHAE over-expression, mock-transduced, and untransduced OVCAR3 and A2780 cells. e, fWestern blot of YWHAE knock-down, mock-transduced, and untransduced CAOV3 and ES2 cells. $\mathbf{g}, \mathbf{h}$ Effects of high YWHAE expression on the invasion of ovarian cancer in OVCAR3 and A2780 cells. $\mathbf{i}$, $\mathbf{j}$ Effects of low YWHAE expression on the invasion of ovarian cancer in CAOV3 and ES2 cells. $\mathbf{k}$, I Effects of high YWHAE expression on the migration of ovarian cancer in OVCAR3 and A2780 cells. $\mathbf{m}, \mathbf{n}$ Effects of low YWHAE expression on the migration of ovarian cancer in CAOV 3 and ES2 cells 


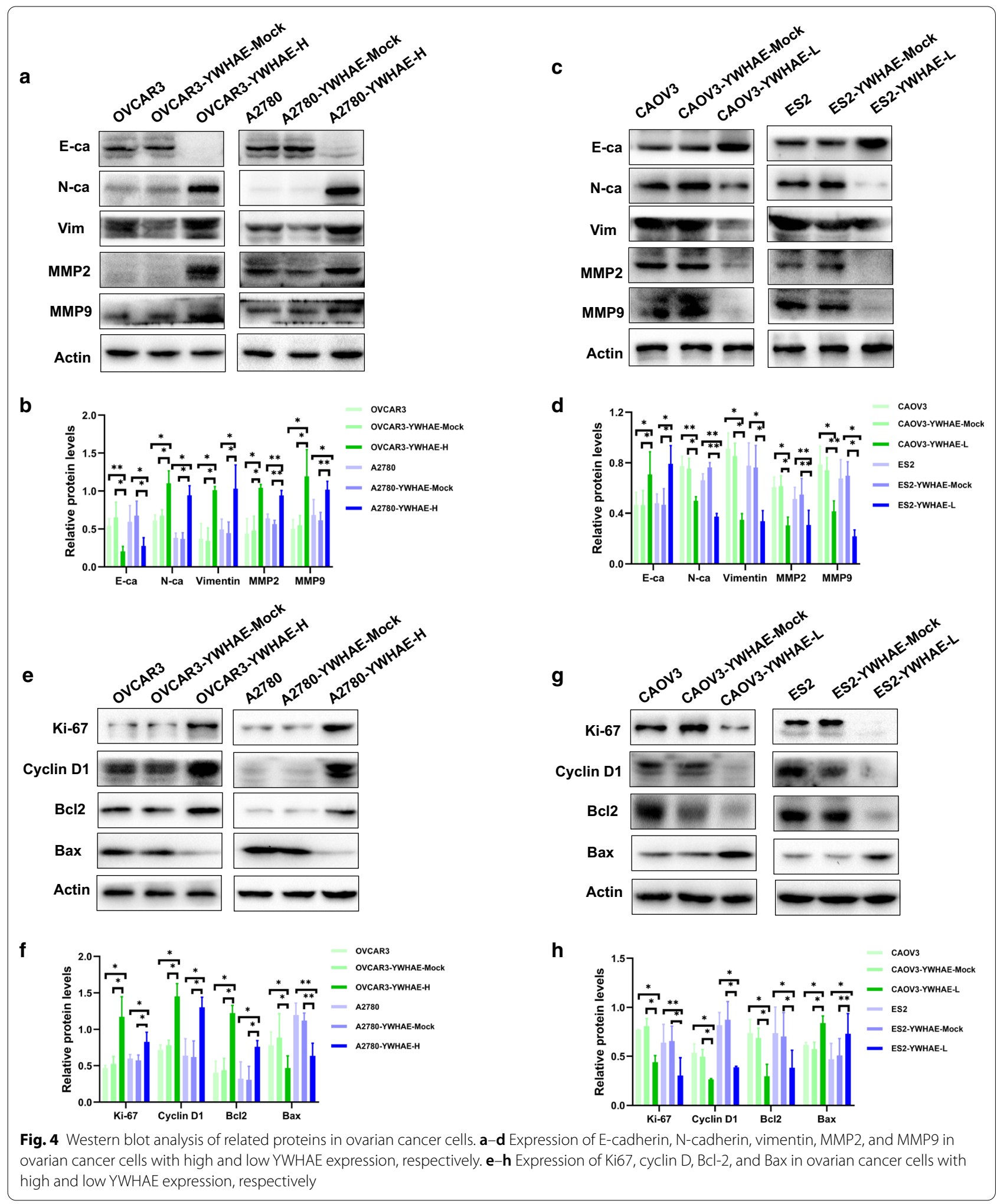




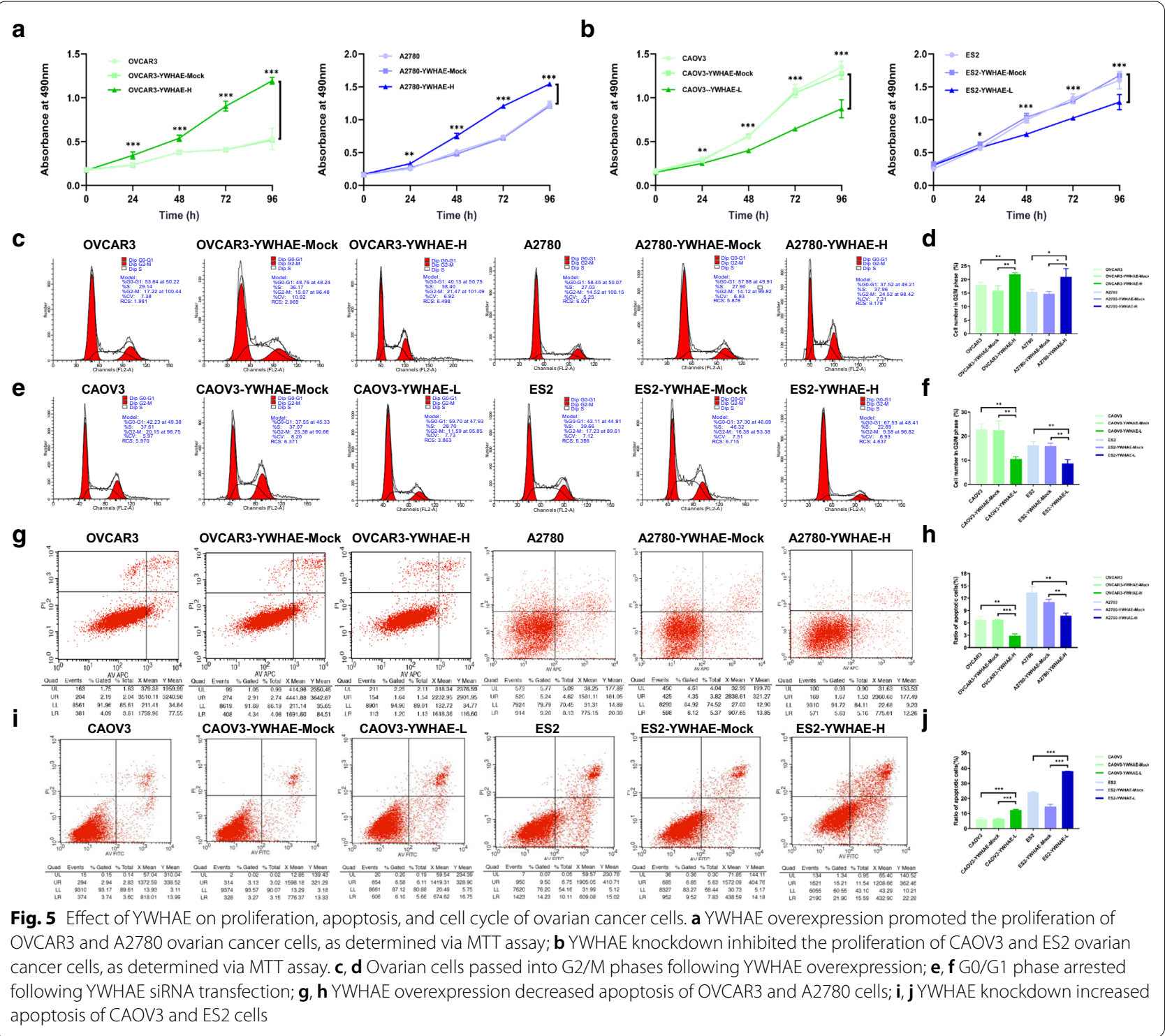

YWHAE, or a mock control, were injected into athymic nude mice. Assessment of the tumours formed 21 days after the cells were injected, revealed that tumours overexpressing YWHAE were significantly bigger, and weighed approximately 2.83 times more than the tumours observed in the control group. Moreover, the growth rate of the tumours produced by YWHAE-overexpressing cells was significantly faster compared with those in the control group (Fig. 6a-c). Immunohistochemistry of tumour biopsies revealed that AKT and ERK (p-AKT and p-ERK, respectively) expression was higher in OVCAR3 cells overexpressing YWHAE, further suggesting that YWHAE can promote the proliferation of tumour cells in vivo (Fig. 6d).
YWHAE-induced cellular effects are mediated by the PI3K/ AKT and MAPK signalling pathways

We used the STRING database to predict relevant YWHAE PPI and showed that YWHAE had direct or indirect interactions with PI3K, MAPK, ACTR1A, and cAMP-dependent protein kinase (Fig. 7a).

For a more detailed perspective on the underlying mechanisms triggered by YWHAE, the levels of several critical signalling molecules were evaluated by western blotting. The results showed that the ratio of $\mathrm{p}-\mathrm{PI} 3 \mathrm{~K} /$ PI3K, p-AKT/AKT, p-m-TOR/mTOR, p-ERK/ERK, and $\mathrm{p}$-MEK/MEK increased significantly in the presence of YWHAE overexpression, but were reduced upon YWHAE knockdown (all $P<0.05$ ). The above results 


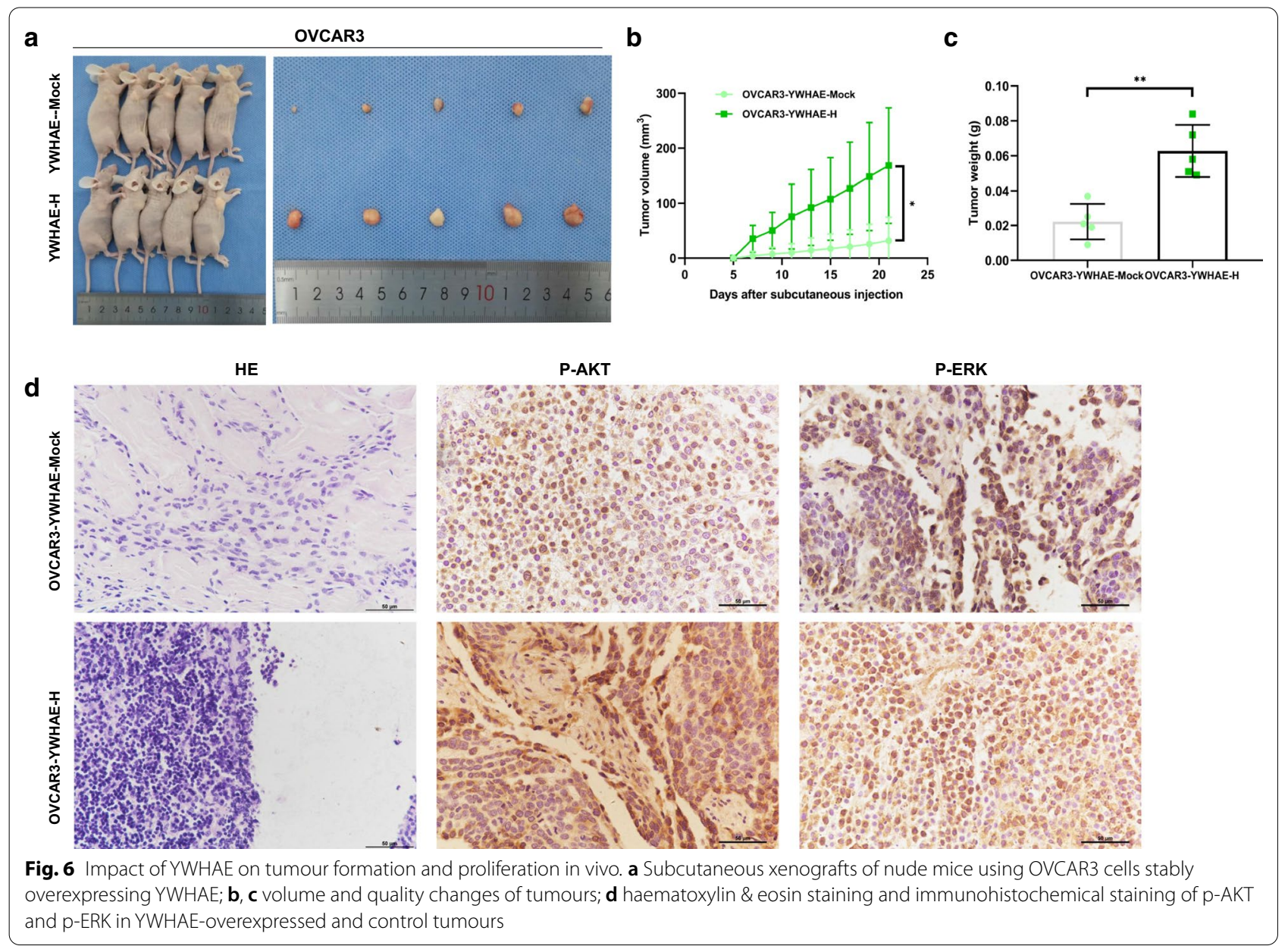

demonstrate that YWHAE can activate PI3K/AKT and MAPK signalling pathways (Fig. 7b-e).

To further explore the role of these two signalling pathways on YWHAE-induced cellular effects, PI3K (GDC-0941) or MEK (PD98059) inhibitors were used. The invasion and migration experiments were repeated in the presence of these inhibitors, revealing that blockage of both PI3K/AKT and MAPK signals significantly weakened the pro-invasion and pro-migration effect promoted by YWHAE overexpression $(P<0.05)$. Moreover, we showed that the proliferation of OVCAR3 cells overexpressing YWHAE was significantly reduced in the presence of GDC-0941 or PD98059 $(P<0.05)$ (Figs. 8, 9).

The above results demonstrate that YWHAE can impact the invasion, migration, and proliferation potentials of ovarian cancer cells, as well as other malignant biological behaviours, through the PI3K/AKT and the MAPK signalling pathways.

\section{Discussion}

Ovarian cancer, a tumour of the female reproductive system, has a high mortality rate. Although a variety of targeted drugs for ovarian cancer are used in the clinical setting, the high mortality rate still represents a serious threat to the lives and health of women worldwide.

The YWHAE protein is widely expressed in eukaryotes, having been detected in wheat [19], giant trematodes in goat blood cells [20], liver flukes [21], and mosquitoes [22]. Moreover, YWHAE has been identified as an important element in human retinal photoreceptor rod cells [23], and is involved in the differentiation of adipose-derived mesenchymal stem cells into osteoblasts, thus enhancing the body's osteogenic ability [24]. In contrast, some studies have detected a peak in YWHAE expression $168 \mathrm{~h}$ following partial liver resection, preventing cell cycle progression and negatively regulating liver regeneration [25]. These discordant findings therefore suggest that YWHAE may have a two-way regulatory effect on the cell cycle. 


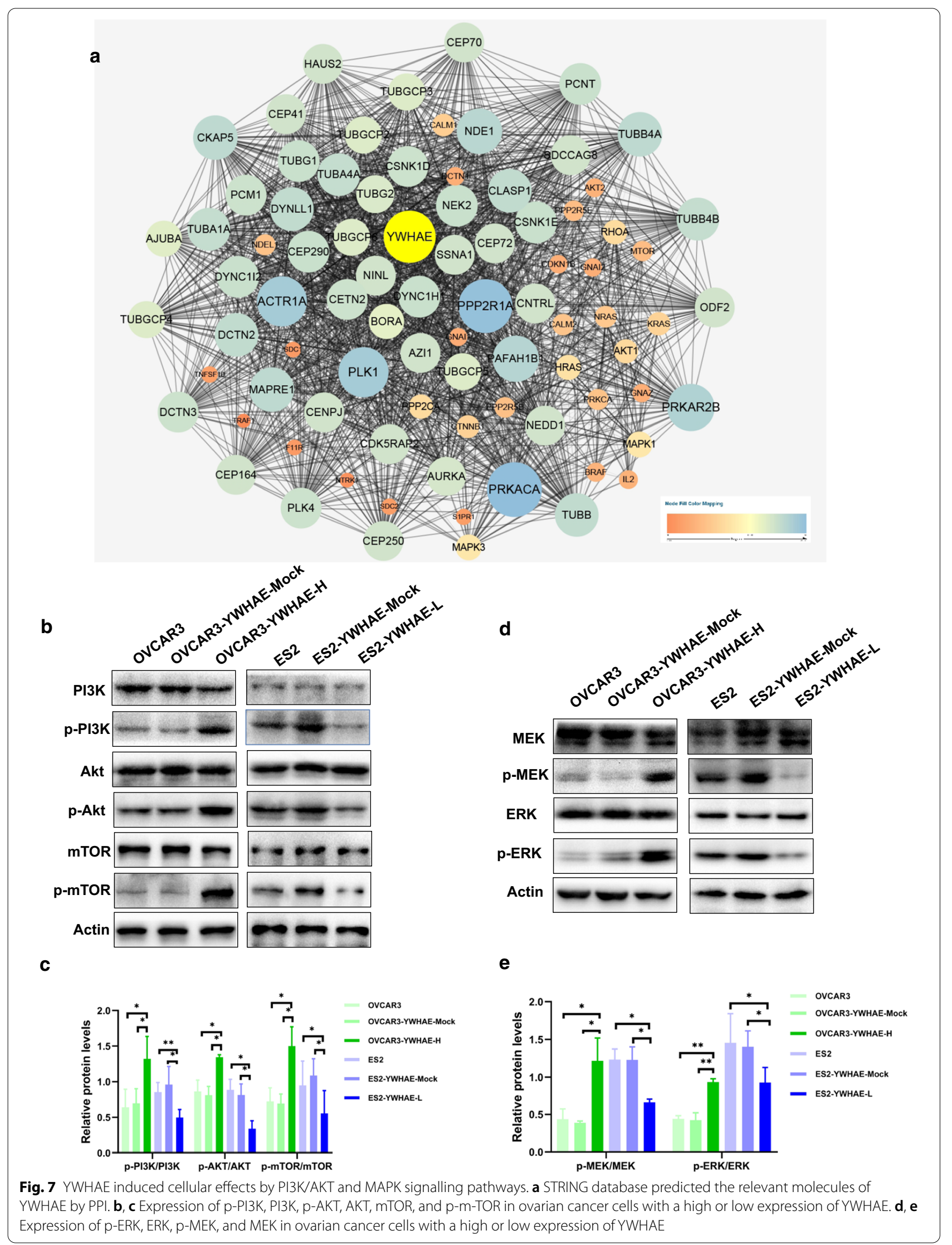




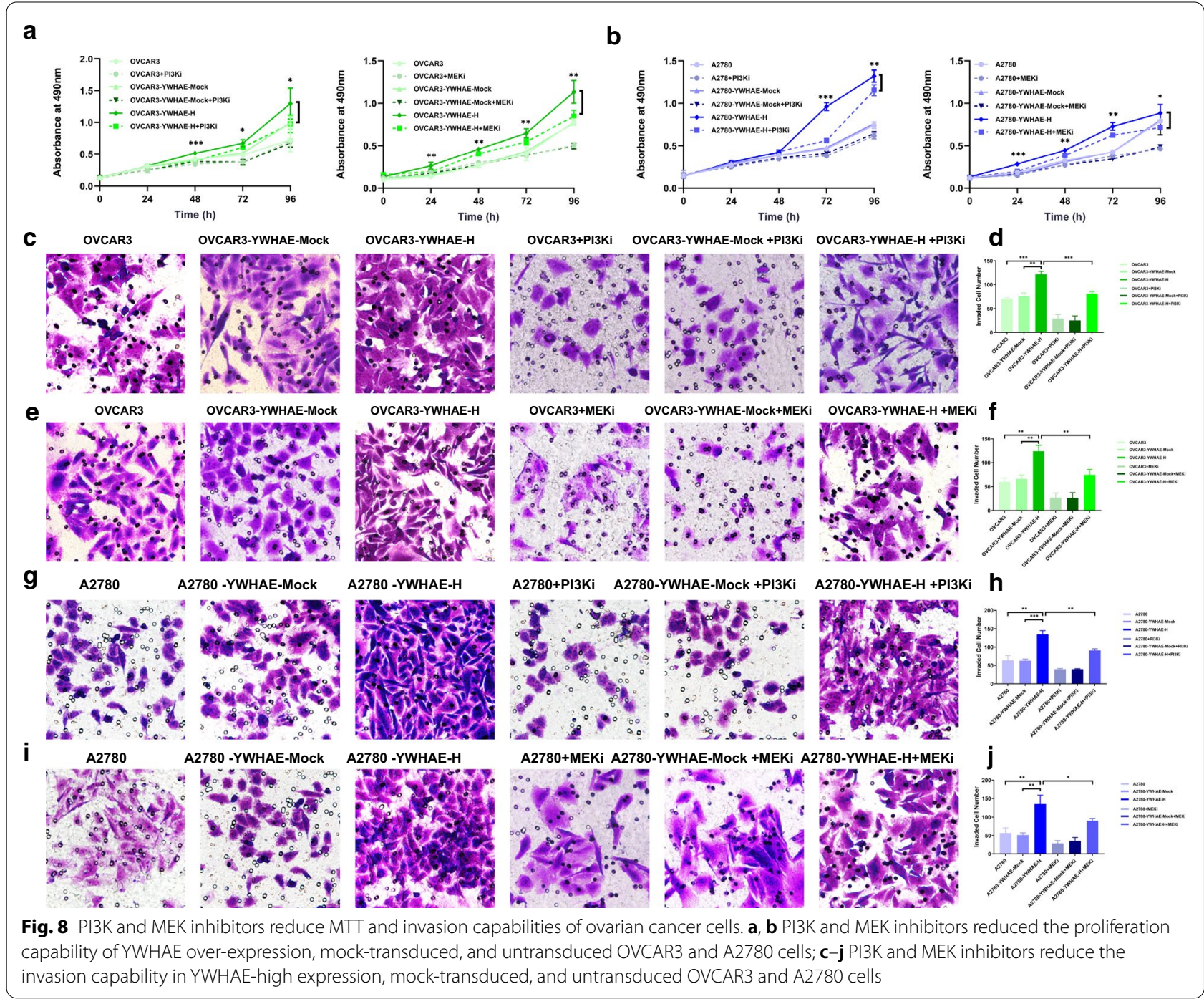

As YWHAE was originally identified in the brain, its pathological effects were initially investigated in the field of neurological diseases, such as Parkinson's [26] and Alzheimer's disease [27], brain excitotoxic injury [28], and myocardial ischemia reperfusion [29], among others. These studies agreed that the mechanism by which YWHAE could impact nerve cells may be related to mitochondrial dysfunction and apoptosis regulation.

In recent years, studies have suggested that abnormal expression of YWHAE may also play an important role in the occurrence and development of tumours. Liang et al. found that YWHAE is highly expressed in kidney cancer tissues, and in vitro experiments demonstrated that YWHAE promotes abnormal proliferation of tumour cells [30]. In gastric cancer cell lines, YWHAE expression is significantly upregulated, and can inhibit cell proliferation, invasion, and migration by reducing the expression of MYC and CDC25B; whereas MYC induces cell proliferation, invasion, and migration by enhancing $\mathrm{CDC} 25 \mathrm{~B}$, and reducing YWHAE expression [12, 31]. In breast cancer, YWHAE expression is related to tumour size, lymph node metastasis, and patient prognosis, as well as breast cancer resistance to chemotherapy. Indeed, YWHAE overexpression significantly increases breast cancer cell proliferation, migration, and invasion, whereas reduced YWHAE expression prevents Snail and Twist expression in breast cancer cells [32]. Although high expression of YWHAE has been described in colorectal, liver, kidney, breast, gastric, and oesophageal cancers, its specific mechanism of action remains unclear.

Among the malignant tumours of the female reproductive system, YWHAE is more commonly reported upon genetic testing of uterine sarcoma cells. Endometrial stromal sarcoma carrying the YWHAE-NUTM2 (or YWHAE-FAM22) fusion gene has obvious malignant biological effects, such as enhanced invasion and 


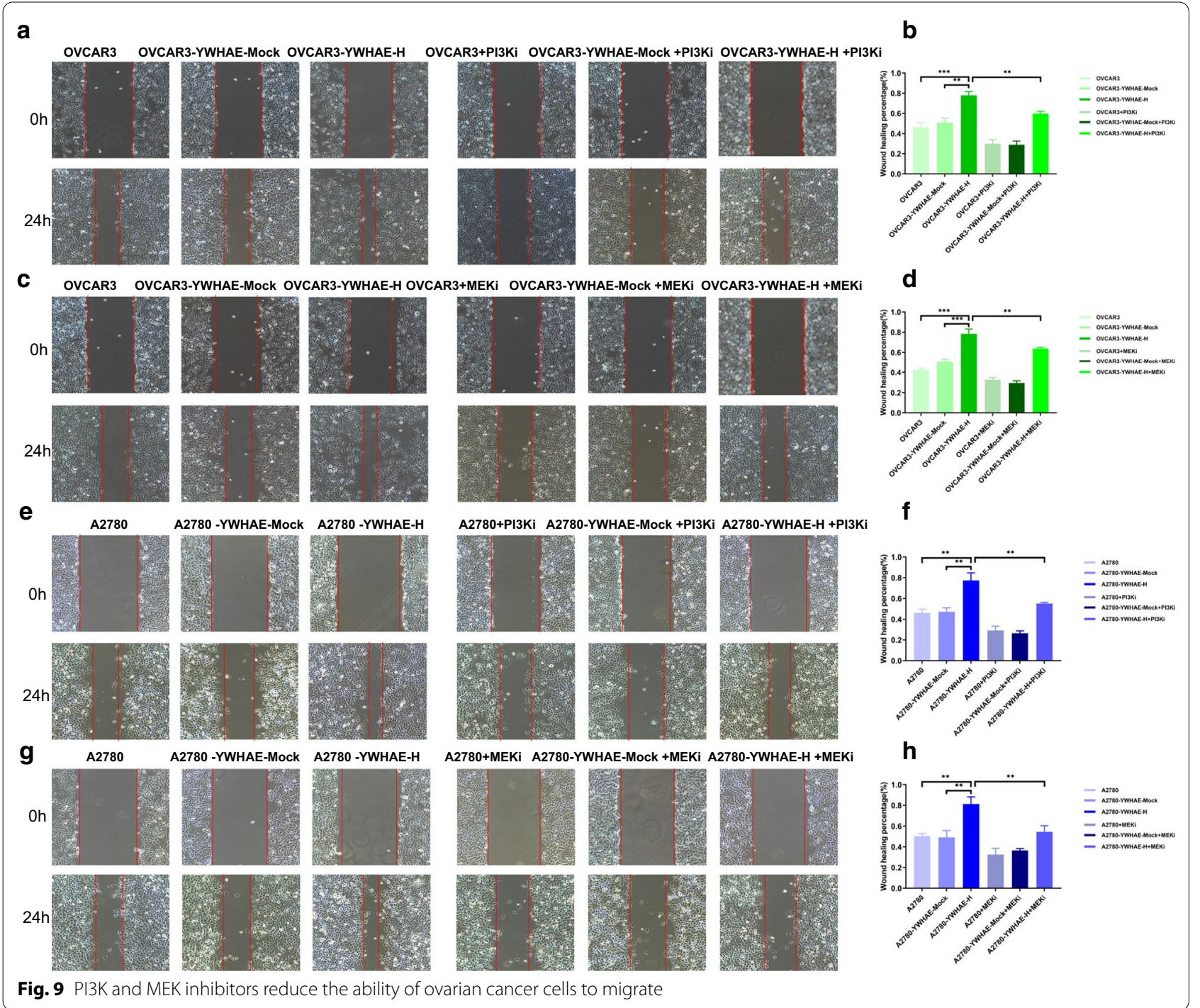

drug resistance. Additionally, the prognosis of patients harbouring such genetic abnormality is poor [33, 34]. Sylvain et al. [35] performed gene chip detection on matched tumour samples from six patients with advanced highgrade epithelial ovarian cancer before and after chemotherapy. The results showed that 54 genes that recurred after chemotherapy were downregulated, whereas 121 genes, including $Y W H A E$, were upregulated. This change in the expression profile suggests that YWHAE may be related to ovarian cancer invasion, proliferation, and drug resistance. Sun et al. used the Gene Expression Omnibus database to analyse the relationship between ovarian cancer and diabetes, and found that 10 key genes, including $Y W H A E$, are important links in the regulation of redox reactions, and carboxylic acid metabolism in the body [36]. Based on these results, they believe that ovarian cancer is related to sugar metabolism, and that certain key metabolism-related genes and proteins could be used as potential targets for the treatment of ovarian cancer.

Taken together, the results described in the present study demonstrate that YWHAE and HE4 are interacting proteins, and that YWHAE is significantly associated with advanced stage cancers and poor patient outcomes. This suggests that high YWHAE expression may represent a risk factor for the prognosis of ovarian cancer. Overall, YWHAE showed a similar cancer-promoting effect as observed for HE4, contributing to the occurrence and development of ovarian cancer.

Through induced differential expression of YWHAE and in vivo experiments, we also demonstrated that YWHAE contributes to ovarian cancer cell invasion, epithelial-mesenchymal transition, and migration, as well as to the enhanced proliferative and anti-apoptotic responses of these cells. 
Previous studies have confirmed that HE4 plays an important role in the metastasis and adhesion of ovarian cancer cells, with low levels of HE4 preventing the activation of ERK and EGFR in ovarian cancer cells. Therefore, it is thought that HE4 may influence the biological behaviour of cancer cells in the ovaries through the EGFR and MAPK signalling pathways; however, the specific underlying mechanism is still unclear. Studies have reported that HE4 can affect the cell cycle (G0/G1 phase), as well as cellular migration and invasion capabilities by regulating ERK/MAPK and the expression of MMP9, MMP2, and cathepsin B [37]. In accordance with its role as an interaction protein of HE4, YWHAE was also shown to affect the malignant biological behaviour of ovarian cancer cells through the abovementioned signalling pathways.

In breast cancer, YWHAEt acts together with 1,3DCQA (eicosanylquinic acid) to prevent the proliferation and metastasis of cancer cells through the Jak/ PI3K/AKT and Raf/ERK pathways, and by inducing the $\mathrm{Bad} / \mathrm{Bax} /$ caspase 9 apoptosis pathway [38]. YWHAE $\zeta$ overexpression can regulate the expression of the protein Snail by activating the PI3K/AKT pathway, thereby promoting the proliferation, migration, and invasion of glioma cells. This protein therefore represents a potential prognostic marker and therapeutic target for glioma [39]. In colorectal cancer, YWHAE $\sigma$ acts as a tumour suppressor gene. However, COPS5 and LASP1, through PI3K/AKT-dependent signalling, stimulate YWHAE $\sigma$ ubiquitination and degradation. This removes the tumour suppressor activity of YWHAE $\sigma$ that promotes the progression of colorectal cancer [40].

Relevant studies have shown that YWHAE can inhibit cell apoptosis in HT-29 colorectal cancer cells [41], and that this process can be reversed by non-steroidal antiinflammatory drugs. The inhibition of apoptosis may be related to the ability of YWHAE to interfere with mitochondrial pro-apoptotic mechanisms, and the activation of transcription factors FKHRL1 and Bad [42]. Moreover, 4-amino-2-trifluoromethyl-phenyl retinoic acid can induce G0/G1 phase arrest in SGC-7901 gastric cancer cells by downregulating YWHAE [43]. We found that PI3K/AKT pathway node proteins (PI3K, $\mathrm{AKT}$, and $\mathrm{mTOR}$ ), and MAPK pathway node proteins (MEK and ERK) were significantly upregulated in ovarian cancer cells overexpressing YWHAE. Importantly, inhibition of these pathways prevented the proinvasion, pro-migration, and pro-proliferation effects induced by YWHAE. Therefore, our results suggest that YWHAE promotes the malignant biological behaviour of epithelial ovarian cancer through activation of the PI3K/AKT and MAPK pathways.

\section{Conclusions}

This study described, for the first time, that YWHAE and HE4 interact and co-localise in cells. We demonstrated that YWHAE expression was significantly increased in ovarian cancer tissues, which was a risk factor for the prognosis of ovarian cancer. Moreover, we showed for the first time that YWHAE could promote the invasion, migration, and proliferation of epithelial ovarian cancer through PI3K/AKT and MAPK pathways. Our results suggest that YWHAE could be used as a prognostic factor and may trigger new research ideas for further understanding the underlying pathogenesis and improving the diagnosis and treatment of ovarian cancer.

\section{Abbreviations \\ HE4: Human epididymis protein 4; FIGO: International Union of Obstetrics and Gynaecology; GSEA: Gene Set Enrichment Analysis; FBS: Foetal bovine serum; DMSO: Dimethyl sulfoxide; FITC: Fluorescein isothiocyanate; DAPI: 4',6-Diamid- ino-2-phenylindole; PBS: Phosphate-buffered saline; PVDF: Polyvinylidene fluoride membrane; KEGG: Kyoto Encyclopedia of Genes and Genomes; GO: Gene Ontology.}

\section{Supplementary Information}

The online version contains supplementary material available at https://doi. org/10.1186/s12935-021-01989-7.

Additional file 1. Verification of three HE4-siRNAs transfection.

\section{Acknowledgements}

This study was supported by the Key Laboratory of Obstetrics and Gynecology of Higher Education of Liaoning Province.

\section{Authors' contributions}

$\mathrm{XL}$ and BL designed the study. CW, SW, YH, SJ, OL, and RG performed the experimental studies. XN and JL performed data analysis, and XL edited the manuscript. All authors read and approved the final manuscript.

\section{Funding}

The study was supported by grants from the National Natural Science Foundation of China (81672590 and 81472437) and an Outstanding Scientific Fund of Shengjing Hospital (201804)

Availability of data and materials Not applicable.

\section{Declarations}

\section{Ethics approval and consent to participate}

The ethics approval and consent to participate was obtained from Shengjing Hospital Affiliated to China Medical University, Shenyang, China (No. 2019PS519K, No. 2019PS520K).

\section{Consent for publication}

The consents for publication from all authors were obtained.

\section{Competing interests}

The authors declare that they have no conflicts of interest.

Received: 6 April 2021 Accepted: 20 May 2021

Published online: 09 June 2021 


\section{References}

1. Wei W, Zeng H, Zheng R, Zhang S, An L, Chen R, et al. Cancer registration in China and its role in cancer prevention and control. Lancet Oncol. 2020;21(7):e342-9.

2. Jelovac $D$, Armstrong DK. Recent progress in the diagnosis and treatment of ovarian cancer. CA Cancer J Clin. 2011;61(3):183-203.

3. Kirchhoff C. Molecular characterization of epididymal proteins. Rev Reprod. 1998;3(2):86-95.

4. Huang J, Chen J, Huang Q. Diagnostic value of HE4 in ovarian cancer: a meta-analysis. Eur J Obstet Gynecol Reprod Biol. 2018;231:35-42.

5. Dochez V, Caillon H, Vaucel E, Dimet J, Winer N, Ducarme G. Biomarkers and algorithms for diagnosis of ovarian cancer: CA125, HE4, RMI and ROMA, a review. J Ovarian Res. 2019:12(1):28.

6. Wang J, Deng L, Zhuang H, Liu J, Liu D, Li X, et al. Interaction of HE4 and ANXA2 exists in various malignant cells-HE4-ANXA2-MMP2 protein complex promotes cell migration. Cancer Cell Int. 2019;19:161.

7. Zhuang H, Tan M, Liu J, Hu Z, Liu D, Gao J, et al. Human epididymis protein 4 in association with Annexin II promotes invasion and metastasis of ovarian cancer cells. Mol Cancer. 2014;13:243.

8. Diallo K, Oppong AK, Lim GE. Can 14-3-3 proteins serve as therapeutic targets for the treatment of metabolic diseases? Pharmacol Res. 2019;139:199-206.

9. Cau Y, Valensin D, Mori M, Draghi S, Botta M. Structure, function, involvement in diseases and targeting of 14-3-3 proteins: an update. Curr Med Chem. 2018;25(1):5-21.

10. Morrison DK. The 14-3-3 proteins: integrators of diverse signaling cues that impact cell fate and cancer development. Trends Cell Biol. 2009;19(1):16-23.

11. Wilker E, Yaffe MB. 14-3-3 Proteins - a focus on cancer and human disease. J Mol Cell Cardiol. 2004;37(3):633-42.

12. Aghazadeh $Y$, Papadopoulos $V$. The role of the 14-3-3 protein family in health, disease, and drug development. Drug Discov Today. 2016;21(2):278-87.

13. Hartman AM, Hirsch AKH. Molecular insight into specific 14-3-3 modulators: inhibitors and stabilisers of protein-protein interactions of 14-3-3. Eur J Med Chem. 2017;136:573-84.

14. Kaplan A, Morquette B, Kroner A, Leong S, Madwar C, Sanz R, et al. Smallmolecule stabilization of 14-3-3 protein-protein interactions stimulates axon regeneration. Neuron. 2017;93(5):1082-93.

15. Jones DH, Ley S, Aitken A. Isoforms of 14-3-3 protein can form homo- and heterodimers in vivo and in vitro: implications for function as adapter proteins. FEBS Lett. 1995;368(1):55-8.

16. Yaffe MB. How do 14-3-3 proteins work? Gatekeeper phosphorylation and the molecular anvil hypothesis. FEBS Lett. 2002;513(1):53-7.

17. Yang X, Qian K. Protein O-GlcNAcylation: emerging mechanisms and functions. Nat Rev Mol Cell Biol. 2017;18(7):452-65.

18. Veisova D, Rezabkova L, Stepanek M, Novotna P, Herman P, Vecer J, et al. The $\mathrm{C}$-terminal segment of yeast $\mathrm{BMH}$ proteins exhibits different structure compared to other 14-3-3 protein isoforms. Biochemistry. 2010;49(18):3853-61.

19. Guo J, Dai S, Li H, Liu A, Liu C, Cheng D, et al. Identification and expression analysis of wheat TaGF14 genes. Front Genet. 2018;9:12.

20. Tian AL, Lu M, Calderón-Mantilla G, Petsalaki E, Dottorini T, Tian X, et al. A recombinant Fasciola gigantica 14-3-3 epsilon protein ( $r F g 14-3-3 e$ ) modulates various functions of goat peripheral blood mononuclear cells. Parasites Vectors. 2018;11(1):152

21. Kafle A, Puchadapirom P, Plumworasawat S, Dontumprai R, Chan-On W Buates $S$, et al. Identification and characterization of protein 14-3-3 in carcinogenic liver fluke Opisthorchis viverrini. Parasitol Int. 2017;66(4):426-31.

22. Trujillo-Ocampo A, Cázares-Raga FE, Del Angel RM, Medina-Ramírez F, Santos-Argumedo L, Rodríguez MH, et al. Participation of $14-3-3 \varepsilon$ and 14-3-32 proteins in the phagocytosis, component of cellular immune response, in Aedes mosquito cell lines. Parasites Vectors. 2017;10(1):362.

23. Inamdar SM, Lankford CK, Laird JG, Novbatova G, Tatro N, Whitmore SS, et al. Analysis of 14-3-3 isoforms expressed in photoreceptors. Exp Eye Res. 2018:170:108-16.

24. Rivero G, Aldana AA, Frontini Lopez YR, Liverani L, Boccacini AR, Bustos DM, et al. 14-3-3e protein-immobilized PCL-HA electrospun scaffolds with enhanced osteogenicity. J Mater Sci Mater Med. 2019;30(9):99.
25. Xue D, Xue Y, Niu Z, Guo X, Xu C. Expression analysis on 14-3-3 proteins in regenerative liver following partial hepatectomy. Genet Mol Biol. 2017:40(4):855-9.

26. Jiang $H$, Yu Y, Liu S, Zhu M, Dong $X$, Wu J, et al. Proteomic study of a Parkinson's disease model of undifferentiated SH-SY5Y cells induced by a proteasome inhibitor. Int J Med Sci. 2019;16(1):84-92.

27. Krzyzanowska A, García-Consuegra I, Pascual C, Antequera D, Ferrer I, Carro E. Expression of regulatory proteins in choroid plexus changes in early stages of Alzheimer disease. J Neuropathol Exp Neurol. 2015;74(4):359-69.

28. Smani D, Sarkar S, Raymick J, Kanungo J, Paule MG, Gu Q. Downregulation of 14-3-3 proteins in a kainic acid-induced neurotoxicity model. Mol Neurobiol. 2018;55(1):122-9.

29. Boyd JG, Smithson LJ, Howes D, Muscedere J, Kawaja MD, Canadian Critical Care Translational Biology Group. Serum proteomics as a strategy to identify novel biomarkers of neurologic recovery after cardiac arrest: a feasibility study. Intensive Care Med Exp. 2016;4(1):9.

30. Liang S, Xu Y, Shen G, Liu Q, Zhao X, Xu Z, et al. Quantitative protein expression profiling of 14-3-3 isoforms in human renal carcinoma shows 14-3-3 epsilon is involved in limitedly increasing renal cell proliferation. Electrophoresis. 2009;30(23):4152-62.

31. Leal MF, Ribeiro HF, Rey JA, Pinto GR, Smith MC, Moreira-Nunes CA, et al. YWHAE silencing induces cell proliferation, invasion and migration through the up-regulation of CDC25B and MYC in gastric cancer cells: new insights about YWHAE role in the tumor development and metastasis process. Oncotarget. 2016;7(51):85393-410.

32. Yang $Y F$, Lee $Y C$, Wang $Y Y$, Wang $C H$, Hou MF, Yuan SF. YWHAE promotes proliferation, metastasis, and chemoresistance in breast cancer cells. Kaohsiung J Med Sci. 2019:35(7):408-16.

33. Ferreira J, Félix A, Lennerz JK, Oliva E. Recent advances in the histological and molecular classification of endometrial stromal neoplasms. Virchows Arch. 2018;473(6):665-78.

34. Cotzia P, Benayed R, Mullaney K, Oliva E, Felix A, Ferreira J, et al. Undifferentiated uterine sarcomas represent under-recognized high-grade endometrial stromal sarcomas. Am J Surg Pathol. 2019;43(5):662-9.

35. L'Espérance S, Popa I, Bachvarova M, Plante M, Patten N, Wu L, et al. Gene expression profiling of paired ovarian tumors obtained prior to and following adjuvant chemotherapy: molecular signatures of chemoresistant tumors. Int J Oncol. 2006;29(1):5-24

36. Sun Y, Xiaoyan $H$, Yun L, Chaoqun $L$, Jialing W, Liu Y, et al. Identification of key candidate genes and pathways for relationship between ovarian cancer and diabetes mellitus using bioinformatical analysis. Asian Pac J Cancer Prev. 2019;20(1):145-55.

37. Zhu YF, Gao GL, Tang SB, Zhang ZD, Huang QS. Effect of WFDC 2 silencing on the proliferation, motility and invasion of human serous ovarian cancer cells in vitro. Asian Pacific J Trop Med. 2013;6(4):265-72.

38. Zhou Y, Fu X, Guan Y, Gong M, He K, Huang B. 1,3-Dicaffeoylquinic acid targeting 14-3-3 tau suppresses human breast cancer cell proliferation and metastasis through IL6/JAK2/PI3K pathway. Biochem Pharmacol. 2020;172:113752.

39. Li J, Xu H, Wang Q, Wang S, Xiong N. 14-3-3 invasion by regulating Snail through the PI3K/AKT signaling. Cancer Med 2019;8(2):783-94

40. Zhou R, Shao Z, Liu J, Zhan W, Gao O Pan Z et al. COPS5 and LASP1 synergistically interact to downregulate 14-3-30 expression and promote colorectal cancer progression via activating PI3K/AKT pathway. Int J Cancer. 2018;142(9):1853-64.

41. Liou JY, Ghelani D, Yeh S, Wu KK. Nonsteroidal anti-inflammatory drugs induce colorectal cancer cell apoptosis by suppressing 14-3-3epsilon. Cancer Res. 2007;67(7):3185-91.

42. Liou JY, Lee S, Ghelani D, Matijevic-Aleksic N, Wu KK. Protection of endothelial survival by peroxisome proliferator-activated receptordelta mediated 14-3-3 upregulation. Arterioscler Thromb Vasc Biol. 2006;26(7):1481-7.

43. Zhao $Y$, Fang $X$, Fang $H$, Feng $Y$, Chen $F$, Xia Q. ATPR-induced $G 0 / G 1$ phase arrest in gastric cancer cells by regulating the binding of $14-3-3 \varepsilon$ and filamin A. Cancer Med. 2018:7(7):3373-84.

\section{Publisher's Note}

Springer Nature remains neutral with regard to jurisdictional claims in published maps and institutional affiliations. 